\title{
A DIFUSÃO DA TELEMEDICINA EM PORTUGAL Dos benefícios prometidos aos riscos de um sistema de cuidados a duas velocidades
}

\author{
Carlo Botrugno \\ "L'altro diritto" RUEBES, Universidade de Florença \\ Joana R. Zózimo \\ "L'altro diritto" RUEBES, Socius-ISEG, Universidade de Lisboa
}

Resumo O objetivo primário deste artigo é analisar a política de implementação da telemedicina, à luz das possíveis repercussões que a sua difusão pode induzir, quer na prática médica quotidiana, quer, mais em geral, na organização do SNS. A análise baseia-se em dados secundários obtidos a partir de atos normativos e relatórios publicados pelo Ministério da Saúde e pela Comissão Europeia, tanto no âmbito específico da e-Health, quanto naquele mais abrangente da inovação tecnológica em saúde. Após avaliar os principais eixos da estratégia pública de promoção da telemedicina, realçamos a necessidade de uma visão crítica que acompanhe a difusão dos serviços à distância na prática médica, a fim de evitar repercussões negativas, entre as quais o aumento da tendência de privatização dos serviços de saúde e o agravamento das desigualdades, com o risco inerente de criar um sistema de cuidados de saúde "a duas velocidades".

Palavras-chave: telemedicina, telemonitorização, tecnologias da informação e comunicação em saúde, saúde em linha, saúde móvel, desigualdades em saúde.

\section{The spread of telemedicine in Portugal: from the promised benefits to the risks of a two-speed healthcare system}

Abstract This article's primary goal is to analyse the policy for implementing telemedicine in the light of the possible repercussions its dissemination may have for both daily medical practice and, in more general terms, for the organisation of the Portuguese National Health Service (SNS). The authors base themselves on secondary data taken from regulations and reports published by the Ministry of Health and the European Commission, both specifically with regard to eHealth, and in relation to the broader subject of technological innovation in the health field. After assessing the main lines of the public strategy for promoting telemedicine, they highlight the desirability of a critical vision to accompany the spread of remote medical services, which is needed to avoid negative repercussions such as an increase in the trend towards the privatisation of health services and a worsening of inequalities, with the inherent danger of creating a "two-speed" healthcare system.

Keywords: telemedicine, telemonitoring, health information and communication technologies, online health, mobile health, health inequalities.

La diffusion de la télémédecine au Portugal: des avantages promis aux risques d'un système médical à deux vitesses

Résumé Le premier objectif de cet article est d'analyser la politique de mise en place de la télémédecine, à la lumière des répercussions que sa diffusion peut avoir, non seulement sur la pratique médicale quotidienne, mais aussi sur l'organisation du système national de santé (SNS) en général. L'analyse se base sur des données secondaires obtenues à partir de textes réglementaires et de rapports publiés par le Ministère de la Santé et par la Commission européenne, dans le domaine spécifique de l'e-Health, mais aussi dans le domaine plus vaste de l'innovation technologique dans la santé. Après avoir évalué les principaux axes de la stratégie publique de promotion de la télémédecine, l'article souligne la nécessité de porter un regard critique à la diffusion des services à distance dans la pratique médicale, afin d'éviter les répercussions négatives, parmi lesquelles l'augmentation de la tendance à la privatisation des services de santé et l'aggravation des inégalités, avec le risque de créer un système de santé "à deux vitesses".

Mots-clés : télémédecine, télésurveillance, technologies de l'information et de la communication dans le domaine de la santé, santé en ligne, inégalités dans le domaine de la santé. 
La difusión de la telemedicina en Portugal: de los beneficios prometidos a los riesgos de un sistema de cuidados a dos velocidades

Resumen El objetivo primordial de este artículo es analizar la política de implementación de la telemedicina, a la luz de las posibles repercusiones que su difusión puede inducir, tanto en la práctica médica cotidiana, como, de manera más general, en la organización del SNS. El análisis se basa en datos secundarios obtenidos a partir de actos normativos e informes publicados por el Ministerio de Salud y por la Comisión Europea, tanto en el ámbito específico de la $e$-Health, como en el ámbito más amplio de la innovación tecnológica en salud. Tras evaluar los principales ejes de la estrategia pública de promoción de la telemedicina, realzamos la necesidad de una visión crítica que acompañe la difusión de los servicios a distancia en la práctica médica, con el fin de evitar repercusiones negativas, como el aumento de la tendencia de privatización de los servicios de salud y el recrudecimiento de las desigualdades, con el riesgo inherente de crear un sistema de cuidados de salud "a dos velocidades".

Palabras-clave: telemedicina, telemonitorización, tecnologías de la información y comunicación en salud, salud en línea, salud móvil, desigualdades en salud.

\section{Introdução}

O termo telemedicina identifica um conjunto de serviços de cuidados em saúde mediados pelas tecnologias da informação e comunicação (TIC), que permitem realizar consultas à distância e trocas de dados, quer entre médico e doente, quer entre diferentes profissionais de saúde. De acordo com uma parte substancial da literatura médica e informática, a utilização destes serviços representa um meio fundamental para melhorar a qualidade das prestações de saúde, incrementar o acesso e, ao mesmo tempo, otimizar e poupar recursos financeiros (e.g. Rubies-Feijoo et al., 2010; Zissman, Lejbkowicz e Miller, 2012). De facto, a telemedicina promete uma revolução na organização dos sistemas de saúde contemporâneos, com a possibilidade de mover o foco principal das intervenções médicas do hospital para o domicílio dos doentes e, deste modo, reduzir as deslocações, bem como os internamentos hospitalares desnecessários (Hein, 2009). ${ }^{1}$

Neste contexto, o objetivo primário deste artigo é analisar a política de implementação dos serviços mediados pelas TIC, destacando as possíveis repercussões que a sua difusão pode induzir, quer na prática médica, quer, mais em geral, na organização do Serviço Nacional de Saúde português (doravante SNS). O artigo resulta de uma análise de dados secundários obtidos a partir de atos normativos e relatórios publicados pelo Ministério da Saúde e pela Comissão Europeia, tanto no âmbito específico da $e$-Health, ${ }^{2}$ quanto naquele mais geral da inovação tecnológica em saúde. Após descrever a origem e as principais caraterísticas da telemedicina (ponto 2), desenha-se o retrato da evolução das orientações europeias e das políticas nacionais que visaram promover a introdução das TIC no SNS (ponto 3).

1 Uma primeira versão deste artigo foi enviada para a revista Sociologia, Problemas e Práticas em julho 2017. O artigo foi aceite em dezembro 2017 sob condição de revisão. A última revisão do artigo foi feita e enviada para a redacção da revista em Maio 2018.

2 Embora a expressão "saúde em linha" seja utilizada em documentos oficiais, utilizaremos o termo inglês $e$-Health, visto que é o mais comum na literatura sobre o tema e é facilmente reconhecido em Portugal, tendo mesmo sido utilizado recentemente no âmbito da Portugal e-HealthSummit, em Março de 2018. 
Subsequentemente, esboça-se uma análise crítica sobre a difusão da telemedicina na prática médica, prestando particular atenção à situação atual do SNS, e tendo em consideração os eixos principais da estratégia de promoção das TIC nos sistemas de saúde contemporâneos, ou seja: a poupança de recursos financeiros, o incremento de qualidade no atendimento e a maior acessibilidade aos serviços de saúde (ponto 4).

Na conclusão, contrariamente às premissas altissonantes que servem de alicerce à promoção desta forma de inovação tecnológica, realça-se como a difusão da telemedicina pode ter repercussões negativas para os sistemas públicos de saúde, entre as quais o aumento da tendência de privatização dos serviços de saúde e o agravamento das desigualdades, com o risco inerente de criar um sistema de cuidados de saúde "a duas velocidades", como descrito por Kaplan e Litewka (2008) e Spoonere Gotlieb (2004).

\section{A evolução da telemedicina e o seu impacto na prática médica}

Apesar de a literatura proporcionar uma grande variedade de definições, uma das mais completas é aquela fornecida pela Organização Mundial da Saúde (OMS), que define a telemedicina como:

The delivery of health care services, where distance is a critical factor, by all health care professionals using information and communication technologies for the exchange of valid information for diagnosis, treatment and prevention of disease and injuries, research and evaluation, and for the continuing education of health care providers, all in the interests of advancing the health of individuals and their communities (OMS, 2010: 9).

Atualmente, pode-se considerar que a telemedicina é uma prática bastante difundida nas seguintes especialidades médicas: (a) a telecardiologia, na qual são incluídos todos os dispositivos que permitem realizar o eletrocardiograma à distância, assim como a telemonitorização dos sinais vitais do doente; $(b)$ a teleneurologia, que consiste na avaliação das funções cerebrais de um doente que sofreu de apoplexia, com o intuito de proporcionar uma intervenção farmacológica ou cirúrgica urgente; (c) a telepsiquiatria, que abrange todas as atividades de educação, formação, diagnóstico e tratamento de doenças psiquiátricas, fornecidas através de videoconferência; $(d)$ a telepatologia, ou seja, a transferência de amostras microscópicas para exame e diagnóstico; (e) a telerreabilitação, que consiste na prestação de tratamentos de reabilitação realizados através de videoconferência; e $(f)$ a telerradiologia, ou seja, a transmissão de imagens radiológicas de uma instituição de saúde (spoke) para um centro de excelência $(h u b)$ que efetua um relatório e o reenvia para o doente (OMS, 2010).

Nos últimos anos, a telemedicina recebeu um novo impulso em concomitância com a ampla difusão dos telemóveis de última geração, que permitem ter acesso a cuidados de saúde não só a partir do domicílio do doente ou dos centros de saúde, mas 
também de qualquer outro lugar. Tal facto determinou o aparecimento de uma nova dimensão, a da saúde móvel (também conhecida como m-Health), que abrange um conjunto de aplicações de software e dispositivos de hardware que permitem tanto realizar medições de sinais vitais e antropométricos (pulso, glicémia, temperatura, gordura corporal, etc.), como medir a frequência e intensidade dos movimentos quotidianos do doente. Por este meio, pode-se obter uma telemonitorização constante do estado dos doentes e, portanto, das próprias doenças. Apesar do facto de estes serviços e aplicações prometerem promover o bem-estar da pessoa, nomeadamente a indivíduos afetados por doenças crónico-degenerativas nos últimos anos uma ampla quantidade de estudos e reflexões críticas - em particular, aquelas desenvolvidas no âmbito dos science and technology studies -, destacou que a difusão destes dispositivos na prática médica pode contribuir para redefinir a relação entre saúde e doença (e.g. Matusitz e Breen, 2007; Lehoux, 2008; Pols, 2012; Mort, Roberts e Callén, 2013; Mort et al., 2013).

A adoção de serviços de telemonitorização, por exemplo, implica uma redistribuição de tarefas, tanto entre profissionais de saúde quanto entre profissionais e doentes (Botrugno, 2019; Mort, Roberts e Callén, 2013). Pelo lado dos doentes remotos, estes têm que lidar com o funcionamento dos dispositivos tecnológicos, mas também de assumir um papel dentro de uma ampla rede de relações e trocas de dados. Esta rede inclui tanto os convencionais atores da clínica moderna (médicos, enfermeiros, cuidadores informais, etc.) quanto alguns novos atores (e.g. fornecedores de dispositivos), com os quais os doentes têm que estabelecer e manter relações de confiança e de colaboração (Pols, 2012). Os doentes também devem lidar com as repercussões sociais que resultam da utilização destes dispositivos na vida diária (Adams e Niezen, 2016). Com efeito, estes dispositivos tecnológicos não são apenas portáteis, mas também projetados para funcionar de forma "ubíqua", podendo aderir ao corpo do doente como uma segunda pele (dispositivos usáveis), ou integrados no seu interior de forma mais ou menos permanente (dispositivos implantáveis). Portanto, a sua utilização pode esbater a linha de distinção entre cuidado de saúde e vida diária, remodelando a relação que os mesmos doentes têm com seus corpos e doenças. Por outro lado, os profissionais de saúde têm que lidar com uma quantidade enorme de dados recolhidos por meio de dispositivos tecnológicos, o que implica um trabalho adicional a fim de envolver os doentes (remotos) numa relação que não pode deixar de ser inspirada em determinadas valências de humanidade, necessárias para uma relação de cuidado de saúde eficaz (Ha e Longnecker, 2010; Cazzullo e Poterzio, 2007).

A nível internacional, a regulação destes dispositivos sofre de um notável atraso (Botrugno, 2018), ficando confinada até hoje a algumas recomendações e boas práticas, como nos Estados Unidos, onde a Food and Drug Administration publicou um documento de orientação sobre a utilização da telemedicina (FDA, 2013); ou na União Europeia, onde a Comissão publicou um Livro Verde (Green Paper on Mobile Health), que reúne o estado da arte da saúde móvel e fornece algumas orientações estratégicas para o seu desenvolvimento (Comissão Europeia, 2014a). No entanto, o desenvolvimento da telemedicina revela-se cada vez mais complexo e variado, o que torna difícil propor uma definição suficientemente abrangente da grande heterogeneidade de dispositivos tecnológicos utilizados, e 
dos serviços já disponíveis para os profissionais de saúde e os doentes. De facto, um estudo recente revelou que já se encontram disponíveis mais de 97.000 apps de saúde para telemóvel, o que perspetiva um mercado de proporções enormes, cujo valor foi estimado num montante próximo dos 26 milhares de milhões de dólares para o ano de 2017 (Research2Guidance, 2013), e muito aliciante para os atores privados na saúde.

\section{Uma agenda europeia para a telemedicina}

Na última década, as instituições da União Europeia (UE) mostraram um notável interesse em relação à utilização das TIC no âmbito médico. Já em 2004, com o primeiro e-Health Action Plan, ${ }^{3}$ a Comissão Europeia expressou grande confiança nos potenciais benefícios dos novos serviços de cuidado à distância. O plano assentava em três orientações principais: (i) aliviar a carga financeira que pesa sobre os sistemas públicos de saúde dos estados-membros da UE, dado o constante incremento de patologias crónico-degenerativas; (ii) promover a mobilidade dos doentes, garantindo assistência aos cidadãos que se encontrassem num outro país da UE, ou que queiram deslocar-se a fim de usufruir de uma prestação de saúde; (iii) aproveitar as possibilidades de inovação tecnológica para estimular o desenvolvimento económico na área da UE. ${ }^{4}$ Entre os objetivos estratégicos fixados pelo Action Plan, encontrava-se também a definição de atividades de suporte para promover a difusão dos modelos de telemedicina. De acordo com a Comissão Europeia:

Até final de 2008, a maioria das organizações de saúde e das regiões de saúde na Europa (comunidades, municípios, freguesias) deve estar em condições de prestar serviços em linha, como teleconsultas (segunda opinião médica), receitas eletrónicas, transferência eletrónica de dossiês, telemonitorização e telecuidados (Comissão Europeia, 2004: 23).

Os pilares desta estratégia foram depois confirmados na Comunicação n. ${ }^{\circ}$ 630/2007, que ligou a promoção das TIC em saúde ao cenário desenhado pelo artigo 152. do Tratado sobre o Funcionamento da União Europeia, que na atuação de todas as suas políticas garante um elevado nível de proteção da saúde dos cidadãos. Neste contexto, a Comissão Europeia confirmou a exigência de sustentar o desenvolvimento e a difusão de tecnologias que pudessem contribuir para aperfeiçoar a gestão dos serviços de saúde. Como reiterado na Comunicação n. ${ }^{-6} 630 / 2007$, esta necessidade resulta da pressão crescente com que têm que lidar os sistemas públicos de saúde dos estados-membros,

3 Cf. Comunicação da Comissão Europeia "Saúde em linha - melhorar os cuidados de saúde para os cidadãos europeus: plano de ação para um espaço europeu da saúde em linha", $\mathrm{COM} / 2004 / 356$, de 30 de abril de 2004

Ibidem, pp. 6-10.

Cf. Comunicação da Comissão Europeia “Juntos para a saúde: uma abordagem estratégica para a UE (2008-2013)", COM/2007/630 de 23 de novembro de 2007. 
decorrente do aumento gradual da esperança média de vida, bem como por efeito de uma mudança mais ampla no teor das expectativas individuais dos doentes, cada vez mais envolvidos na gestão do seu estado de saúde, e cada vez menos "objetos passivos" de tratamentos médicos. ${ }^{6}$

Em 2008, a Comissão Europeia publicou a Comunicação n. ${ }^{\circ}$ 689/2008, ${ }^{7}$ o primeiro documento exclusivamente dedicado à prática da telemedicina. Mais uma vez, a Comissão confirmou que o objetivo principal era apoiar os estados-membros no esforço de integrar serviços inovadores de cuidados de saúde mediados pelas TIC, promovendo o conhecimento e a confiança dos cidadãos no seu funcionamento e, portanto, a sua aceitação. ${ }^{8}$

A estratégia da União Europeia nesta área foi renovada no e-Health Action Plan de 2012, ${ }^{9}$ o qual assumiu entre as suas finalidades a de lidar com, e remover, os principais obstáculos que impediam a consolidação desta dimensão de tratamento como uma prática de rotina nos sistemas públicos de saúde dos estados-membros. Neste contexto, a Comissão pediu, aos governos nacionais e aos investidores privados do setor, um esforço conjunto que visasse estimular a consciência e a confiança nas soluções de $e$-Health e mobile-Health entre cidadãos e profissionais de saúde, que encorajasse a investigação e a colaboração internacional nesta área, e ainda que promovesse a interoperabilidade dos modelos utilizados nos diferentes níveis dos sistemas de saúde e nos diferentes países. ${ }^{10}$

\section{A promoção da telemedicina em Portugal}

O interesse na telemedicina em Portugal remonta a 1999, quando foi criada a Comissão de Acompanhamento da Iniciativa Estratégica para o Desenvolvimento da Telemedicina (CIEDT). A vontade de conferir à telemedicina um papel fundamental na reorganização dos cuidados de saúde foi-se destacando em vários documentos governativos; ${ }^{11}$ contudo, somente através da Portaria n. ${ }^{-567 / 2006}{ }^{12}$ se começaram a regulamentar pela primeira vez as tabelas de preços das prestações e serviços de telemedicina dentro do contexto mais geral das prestações forneci-

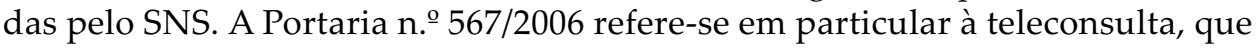
define como:

utilização de comunicações interativas, audiovisuais e de dados em consulta médica com a presença do doente, a qual utiliza estes meios para obter parecer à distância de,

Ibidem, p. 10.

7 Cf. Comunicação da Comissão Europeia "Sobre os benefícios da telemedicina para os doentes, os sistemas de saúde e a sociedade", COM/2008/689 de 4 de novembro de 2008.

$8 \quad$ Ibidem, p. 2.

9 Cf. Comunicação da Comissão Europeia "Plano de ação para a saúde em linha, 2012-2020 Cuidados de saúde inovadores para o século XXI", COM/2012/736, de 6 de novembro de 2012.

10 Ibidem, p. 6

11 Cf. Programa Operacional Saúde XXI (2000) e Programa Operacional Sociedade da Informação (2000).

12 Cf. Portaria n.ำ 567, de 12 de junho de 2006, publicada no Diário da República, n.ำ113, I série-B. 
pelo menos, outro médico e com registo obrigatório no equipamento e no processo

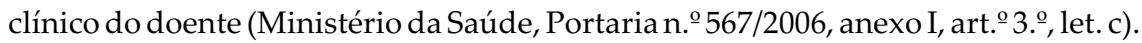

Na Portaria n.․5 567/2006 destacava-se a obrigatoriedade de registar a prestação no processo clínico do doente, o que visava promover a consolidação da estrutura tecnológica de partilha de dados de saúde em linha. A portaria estabelecia ainda que as teleconsultas fossem faturadas pelo mesmo valor das consultas médicas convencionais (i.e. ao vivo), com a possibilidade de acrescentar o valor dos meios auxiliares utilizados..$^{13}$

Atualmente, o principal documento normativo dedicado à telemedicina é o Despacho do secretário de estado adjunto do ministro da Saúde, n.. 3571/2013,14 por meio do qual se tenta esboçar uma estratégia de implementação dos serviços à distância dentro do SNS. De acordo com este despacho, a telemedicina representa uma "ferramenta" à disposição dos profissionais de saúde, que apresenta diversas vantagens. Entre estas, a possibilidade de reduzir a distância física entre serviços de saúde e doentes, tanto como a de evitar deslocações desnecessárias e proporcionar uma maior rapidez no atendimento em algumas especialidades. Conforme o afirmado, a teleconsulta "aumenta a acessibilidade às consultas de Especialidades Médicas, aumenta a equidade, proporcionando a possibilidade de todos os utentes receberem a melhor qualidade de cuidados de saúde, reduz os custos associados" (Despacho n.․ 3571/2013: 8325). Da mesma forma, também à telemonitorização se atribui um papel fundamental para uma gestão mais eficiente das doenças crónicas. Neste contexto, sugere-se a implementação de um sistema interligado com o SNS, e que tenha como objetivo garantir "a monitorização remota, praticada por uma equipa de profissionais de saúde, a partir de uma Instituição sobre um grupo de doentes crónicos que seguem um protocolo a partir dos seus domicílios" (Despacho n. ${ }^{\circ}$ 3571/2013: 8326).

De outro ponto de vista, constatando a falta de uma estratégia coerente para a "massificação" da telemedicina, o Ministério da Saúde considerou prioritário delinear medidas concretas para a instituição de uma Rede de Telemedicina no SNS. Com base nesta perspetiva, estabeleceu que as instituições hospitalares do SNS tinham que implementar a utilização destes serviços em articulação com os Agrupamentos de Centros de Saúde (ACES) da própria área de influência. Por conseguinte, o despacho chegou a identificar as especialidades médicas consideradas como áreas de implementação prioritárias dos serviços de telemedicina: dermatologia, fisiatria, neurologia, cardiologia, cardiologia pediátrica, pneumologia (Despacho n.o 3571/2013: 8326).

O mesmo despacho estabelece ainda que as teleconsultas devem, sempre que possível, ser realizadas em tempo real, e recomenda que pelo menos o primeiro contacto entre o profissional de saúde e o doente ocorra de forma presencial. Esta indicação está em conformidade com uma consistente literatura internacional que sugere uma combinação de encontros ao vivo com encontros virtuais ou, pelo menos, um

13 Ibidem, art. $\stackrel{\circ}{15}$..

14 Cf. Despacho do ministro da Saúde, n. ${ }^{\circ}$ 3571, de 6 de março de 2013, publicado no Diário da República, n. ${ }^{\circ}$ 46, II série. 
primeiro contacto ao vivo, de modo a conciliar as vantagens proporcionadas pela utilização das TIC com a necessidade de proteger a qualidade da comunicação entre médico e doente (e.g. Lindberg et al., 2013; Birns, Bhalla e Rudd, 2010). O Despacho n. - 3571/2013 também se refere ao nível de adequação dos equipamentos tecnológicos utilizados para a prestação dos serviços de saúde à distância, determinando que a contratualização destes seja feita pelas entidades hospitalares que dispõem dos meios tecnológicos necessários.

Por fim, visando traduzir as orientações do Ministério da Saúde numa efetiva política de implementação, o Despacho n. ${ }^{\circ}$ 3571/2013 estabelece também que as Administrações Regionais de Saúde (ARS) apresentem planos regionais para o desenvolvimento da telemedicina em articulação com as entidades hospitalares e com a Comissão para a Informatização Clínica (Despacho n.ำ 3571/2013: 8326).

De acordo com os dados mais recentes, Portugal conta com 160 plataformas de telemedicina, contudo apenas 76 delas foram identificadas como ativas (Matos et al., 2014: 45). Entre as iniciativas mais importantes já realizadas neste campo a nível nacional, assinalam-se: os serviços de telerrastreio dermatológico, que preveem a possibilidade de um médico de medicina geral anexar imagens de relevância dermatológica e enviá-las a um médico dermatologista, obtendo por meio telemático uma indicação sobre a situação clínica do doente; as teleconsultas de especialidade, implementadas a partir de 2015 através de o sistema "PDS-Live", o que constitui um desenvolvimento do sistema de Plataforma de Dados de Saúde (PDS), já ativo no SNS; e os serviços de telemonitorização domiciliária, que contam com vários projetos experimentais ou quase experimentais que visam controlar remotamente o estado de saúde dos doentes crónicos. ${ }^{15}$

\section{Poupança, qualidade e acessibilidade: dos benefícios prometidos aos riscos da telemedicina}

Nesta secção esboçar-se-á uma avaliação dos maiores benefícios e riscos de implementação da telemedicina na prática médica, dando particular atenção à situação do SNS. Tomamos como referência os três maiores pilares da estratégia de promoção da telemedicina, resultantes das orientações adotadas pela UE nas últimas duas décadas (Comissão Europeia, 2004, 2007, 2008, 2012): (i) a poupança de recursos financeiros; (ii) o incremento de qualidade no atendimento; (iii) a maior acessibilidade aos serviços de saúde. Estes não são escolhidos apenas por serem importantes referências supranacionais, mas também porque se enquadram na tendência atual de modernização da gestão pública, através de medidas de matriz racional e legal, a que Portugal não é alheio (cf. Correia e Denis, 2016). De facto, este tipo de orientações facilitaram novos modelos de governação da saúde, em que valores tipicamente associados à área de

15 Entre os quais se assinalam um projeto de telemonitorização de doentes com doença pulmonar crónica obstrutiva, desenvolvido em parceria com os hospitais de Viana do Castelo, Covilhã, Coimbra, Évora e Faro, e um projeto de telemonitorização de doentes cardíacos entre o Hospital Universitário dos Covões e o Hospital Universitário de Coimbra. 
gestão - processo que tem vindo a ser denominado "managerialismo" -, com tónica no uso eficiente dos recursos, na inovação administrativa e na qualidade do desempenho, se aliam a valores característicos da profissão médica na gestão de serviços e prestação de cuidados de saúde (Correia, 2013; Kuhlmann et al., 2013; Correia e Denis, 2016) como, entre outros, a telemedicina.

No caso desta, a oportunidade de poupar recursos em saúde é associada à redução significativa das admissões hospitalares, que seria obtida por meio da integração de teleconsultas e telemonitorização nos cuidados convencionais. Porém, no contexto dos sistemas de saúde dos países industrializados, uma eventual redução do volume de internamentos hospitalares, por si só, pode não levar automaticamente a um ganho de recursos financeiros. Como realçado pragmaticamente pela literatura (Blunt, Bardsley e Dixon, 2010; Gornall, 2012), as vagas deixadas livres pelos doentes telemonitorizados seriam ocupadas pelos demais doentes em lista de espera, cuja diminuição é sensível a vários outros fatores, como os ciclos políticos e epidemiológicos (cf. OPSS, 2017). Assim, pode-se duvidar do potencial da telemedicina em reduzir estas listas, embora seja um objetivo notável no âmbito de uma política que vise ampliar a acessibilidade aos serviços públicos de saúde. Todavia, além de ser uma hipótese que carece de verificação, é difícil entender como a introdução das TIC possa levar a poupanças significativas nas despesas em saúde, tanto para o SNS quanto para os doentes. No caso da teleconsulta de especialidade, por exemplo, é sempre necessário ter um médico, assim como cobrar o valor da prestação. Para além disso, a estes "custos fixos" deve-se acrescentar o valor de aquisição e manutenção dos novos equipamentos tecnológicos necessários à realização das prestações à distância, quer sejam disponibilizados pelo SNS ou por empresas privadas.

Portanto, há um dado que parece inegável: a implementação da telemedicina comporta um aumento dos custos relativamente às prestações convencionais, tal como demonstrado por uma ampla literatura (e.g. Chaudhry et al., 2010; Steventon e Bardsley, 2012). Neste contexto, o sistema "PDS-Live" destaca-se como um exemplo virtuoso, por ser uma implementação tecnológica "simples" e de baixo custo, que integra uma câmara web com um sistema de partilha de dados já em funcionamento no SNS (a Plataforma de Dados de Saúde - PDS).

Porém, aqui o problema passa da conveniência económica dos serviços de telemedicina à qualidade do cuidado que permitem alcançar, o que representa o segundo eixo da nossa análise. Em termos gerais, poder-se-ia afirmar que a telemedicina aumenta a qualidade dos serviços de saúde, sobretudo se não houver um "efeito de substituição", ou seja, enquanto a prestação convencional não desaparecer "por detrás" da prestação virtual. Consideremos, por exemplo, uma teleconsulta de especialidade com intuito de obter uma segunda opinião: recorrer à opinião de mais um especialista via telemedicina, depois de ter consultado um primeiro especialista ao vivo, poderia contribuir para lidar com aquelas situações em que é mais difícil obter um diagnóstico preciso. A questão complica-se nos casos em que a telemedicina é proposta para substituir os serviços de cuidado convencionais. Uma parte notável da literatura sobre a telemedicina (e.g. Bergrath et al., 2011; Gregoski et al., 2012) tem subestimado as implicações da interação virtual entre médico e doente, tendendo a propor uma assimilação imprudente entre a mera viabilidade tecnológica das 
ligações telemáticas e a aceitabilidade clínica e social dos cuidados que por meio delas são providenciados. Com efeito, na literatura que subscreve esta perspetiva, o foco da relação clínica muda significativamente para a análise de dados, e o doente é convertido num conjunto de representações visuais e informações digitais transmitido telematicamente e analisado em sua ausência, o que pode contribuir para incrementar a vocação reducionista da biomedicina (Webster, 2002; Raposo, 2009).

Vale a pena lembrar que, nas últimas duas décadas, vários autores destacaram o perigo de a difusão dos serviços de telemedicina induzir uma nova forma de "desumanização" em saúde (e.g. Bauer, 2001; Leh, 2001; Roback e Herzog, 2003; Zuiderent, Berg e Winthereik, 2003; Matusitz e Breen, 2007). Visando proteger o valor das conexões estabelecidas na relação entre profissional de saúde e doente, assim como compensar os efeitos do distanciamento induzido pela mediação tecnológica, a literatura mais atenta tem realçado a necessidade de combinar as ligações e trocas de dados telemáticas com uma série de encontros ao vivo (Lindberg et al., 2013; Steventon e Bardsley, 2012; Birns, Bhalla e Rudd, 2010; Botsis e Hartvigsen, 2008). Esta indicação é conforme ao estabelecido pelo Ministério da Saúde, que recomenda que as teleconsultas ocorram após o profissional de saúde e o doente terem tido um contacto prévio presencial (Despacho n.. 3571/2013: 8326).

Deve-se reservar uma atenção ainda maior para a questão da telemonitorização de pessoas afetadas por doenças crónico-degenerativas. Neste âmbito, pode-se afirmar que o doente que reúne condições para ser telemonitorizado em exclusivo se encontra geralmente numa situação ainda não incapacitante, ou seja, trata-se de um doente que conduz uma vida "ordinária", mas com algumas limitações decorrentes dos riscos de agravamento do seu estado de saúde. Telemonitorizar este doente significa dar-lhe mais segurança na sua vida diária, aumentando a frequência de contactos telemáticos com profissionais de saúde (Nicastri, 2012), mas também lembrar-lhe quotidianamente a sua condição de doente, quer nas relações sociais, quer no modo como se percebe a si mesmo (Mort, Roberts e Callén, 2013), o que pode ter repercussões negativas a nível psicossocial. Entre estas, assinala-se, em particular, o potencial surgimento ou incremento de ansiedade e sensação de isolamento, em parte também devido à diminuição das relações interpessoais com os profissionais de saúde, em relação com um aumento de contactos digitais (Cartwright et al., 2013).

Além do exposto, é ainda importante destacar que a difusão destes serviços na prática médica parece basear-se numa nova exigência, a de ter "mais segurança" na gestão do estado de saúde dos doentes crónicos (Comissão Europeia, 2004, 2014a). Ou seja, resulta da necessidade de os profissionais de saúde terem acesso a um maior número de dados, mais atualizados, o que é proporcionado por uma maior frequência de contactos telemáticos do que com os serviços convencionais. A defesa desta segurança coloca, pelo menos, dois novos problemas: ao nível da autonomia do paciente, e ao nível da privatização da saúde.

Primeiro, os cuidados convencionais, ao dependerem mais da informação prestada pelo doente e do seu trabalho quotidiano de gestão da doença crónica (Baszanger, 1986), tinham-se tornado um terreno propício a uma maior negociação do trabalho médico, à valorização da experiência do doente, e à promoção da sua 
autonomia. De acordo com a "visão inovadora" que acompanha a política de implementação da telemedicina, os serviços à distância permitem aos doentes remotos serem cada vez mais "engajados" na relação de cuidado e confortados pela frequência de contactos que podem ter, ainda que em muitos casos estes contactos sejam estabelecidos com interfaces tecnológicas (semi)automáticas. Sem dúvida, a promoção de uma maior autonomia dos doentes, sobretudo aqueles que sofrem de doenças crónicas, representa um objetivo notável. Contudo, no debate recente sobre a telemonitorização, esta visão particular de autonomia tem sido criticada por se inspirar numa noção política do ser humano:

This concept projects the individual as being independent and self-sufficient and views relationships as secondary, fashioning these relationships in more or less contractual terms where people act as equal citizens within a public realm. This political notion of autonomy assumes that people are individuals who make decisions for and about themselves and are hence self-determining. The specific ethical realm of (care) relationships, however, is characterised by asymmetry, vulnerability and dependency (Zwijsen, Niemeijer e Hertogh, 2011: 425).

A telemonitorização recomplexifica a distribuição de poder entre médico e doente, pois implica uma "ativação digital" deste (Lupton, 2013), que pode afinal contribuir para que a maior autonomia proporcionada (pelo menos em abstrato) pelos serviços remotos redunde numa "hiper-responsabilização" do mesmo (Kaplan e Litewka, 2008). Refutando a visão que representa o doente remoto como "mais autónomo" do que aquele assistido em formato convencional, vários autores destacam como o constrangimento incutido nos utentes pela utilização diária de dispositivos tecnológicos pode ultrapassar a promoção de uma efetiva autonomia (Chiapperino et al., 2012; Nagel e Remmers, 2012) - arriscando tornar-se uma nova forma de paternalismo e de opressão tecnológica. Paralelamente, estudos empíricos realçaram que, em muitos casos, o perseguir deste objetivo parece corresponder mais a lógicas direcionadas a reduzir os custos das despesas em saúde do que a incrementar a qualidade dos cuidados prestados (Milligan, Roberts e Mort, 2011; Lupton, 2013; Mort, Roberts e Callén, 2013).

Além disso, embora se possa especular em que medida a exigência de maior segurança é prioritária - ou, pelo menos, mais do que outras, à luz do contexto atual de escassez de recursos em saúde -, é inegável que alimenta um mercado interminável de novas prestações, o que se enquadra nos interesses das empresas privadas que fornecem estes serviços inovadores e promovem a manutenção das equipas tecnológicas. Esta possibilidade ganha maior impacto quando contextualizada à luz de um SNS que, em Portugal, é já pautado por uma convivência entre serviço público e privado que pode ser nociva para os interesses dos cidadãos (Santos e Ferreira, 2002; Hespanha, 2008), agravada nos últimos anos de políticas anticrise:

É certo que a tendência para substituir a produção direta de serviços públicos por prestação indireta através de prestadores privados (não-lucrativos ou mesmo lucrativos) já se verificava em Portugal muito antes da crise (Hespanha, 2008). 
Mas tem sido crescente o uso do argumento do excesso de burocracia estatal para o Governo impor uma agenda de transferência de responsabilidades para a esfera privada (Hespanha, Ferreira e Pacheco, 2014: 211-212).

Deste modo, o fornecimento das tecnologias para prestação de serviços de saúde públicos por parte de privados permite-nos não só temer uma maior promiscuidade entre serviços públicos e privados, mas também retomar as dúvidas quanto ao potencial de poupança da telemedicina. O aumento de custos para o SNS relacionado com a dependência tecnológica em saúde e com modelos de financiamento não públicos é demonstrado por extensa literatura (cf. Quesnel-Vallée et al., 2012; OECD/EU, 2014; Correia et al., 2015), e leva-nos assim ao último ponto de análise dos argumentos que têm suportado a esperança na $e$-Health: as desigualdades no acesso a cuidados de saúde.

No que diz respeito à melhoria da acessibilidade, pelo menos em abstrato, a difusão da telemedicina permitiria mais possibilidades de aceder aos serviços de saúde. Contudo, é preciso ter em conta duas ordens de fatores. Primeiro, o SNS é regionalmente assimétrico (Cortes, 2016), sendo os territórios com menor cobertura de serviços de saúde - as áreas periféricas, rurais ou distantes dos grandes centros urbanos - aqueles em que as populações têm mais dificuldades em dispor de recursos tecnológicos (EuroHealthNet, 2011; Comissão Europeia, 2013). Segundo, os grupos em maior desvantagem ${ }^{16}$ no acesso aos serviços de saúde $-e . g$. cidadãos/ãs incapacitados/as; que vivem em condição de isolamento social e/ou geográfico, ou em piores condições económicas e sociais (cf. OMS/CSDH, 2003; Lindsay et al., 2008; Cortes, 2016; McAuley, 2014; Kontos et al., 2014; Latulippe, Hamel e Giroux, 2017), são também os que dispõem de menos tecnologia e de menos conhecimentos para lidar com o seu funcionamento (Puga et al., 2009), o que reforça a ideia de que serão também os menos beneficiados pelo advento da telemedicina.

À luz destas considerações, pode-se prever que quem mais beneficiaria das oportunidades oferecidas pela difusão da telemedicina seriam os que têm capacidades e recursos suficientes para conjugar as prestações convencionais com os serviços mediados à distância, visto que se encontram já em vantagem tecnológica e socioeconómica. Isto leva a concluir que a estratégia pública para a promoção da telemedicina deve abandonar a "retórica da acessibilidade", e focar-se na elaboração de medidas concretas que visem a superação - ou pelo menos a diminuição - dos digital divides em saúde, e a promoção de uma efetiva e-Health literacy ${ }^{17}$ (Comissão Europeia, 2014b).

Concluindo, as mais recentes avaliações do SNS (cf. Correia et al., 2015; Hespanha, 2017; OPSS, 2017) caracterizam-no como ainda em recuperação da crise de

16 Estes grupos são expressamente mencionados no âmbito da recomendação adotada pelo Conselho da Europa em 2007, sobre as medidas para promover o valor público da internet (Rec. Conselho de Europa, n.․ำ 2007/16, II).

17 A e-Health literacy foi definida como: "the ability to seek, find, understand, and appraise health information from electronic sources and apply the knowledge gained to addressing or solving a health problem" (Norman e Skinner, 2006: 2). 
2008, durante a qual houve um aumento das listas de espera, a diminuição do número de profissionais, o agravamento das desigualdades sociais, a manutenção do desequilíbrio entre cuidados hospitalares e primários, a dependência acrescida sobre os cuidadores familiares, entre outros fatores que colocam pressão sobre o sistema de saúde, mais do que a modalidade (presencial ou remota) em que é prestado o serviço. Pelo exposto anteriormente, é dúbio que a telemedicina contribua para atenuar estas dificuldades, se é que não contribui mesmo para as aprofundar.

\section{Conclusões. A telemedicina e o risco de um sistema de cuidados de saúde a duas velocidades}

A visão inovadora associada à promoção da telemedicina, que resulta da análise das orientações adotadas pela UE e pelo Ministério de Saúde, parece conter várias contradições. Por um lado, a difusão dos serviços à distância promete reduzir os custos, evitar duplicações de exames e limitar as admissões hospitalares. Por outro lado, ela inspira-se numa "lógica incremental", que associa melhores cuidados de saúde à possibilidade de aceder a um número cada vez maior de serviços (remotos). Com efeito, apesar de este acesso se tornar cada vez mais frequente, as prestações à distância resultam em relações mais fragmentadas e desumanizadas - ou mecanizadas, se assim se preferir - como é no caso da telemonitorização, onde um sistema calibrado com base em alguns parâmetros predeterminados "controla" o estado de saúde do doente-utente, e emite sinais de alerta caso este varie significativamente.

À luz das considerações expostas anteriormente, outro risco que acompanha a difusão da telemedicina na prática médica é que ela possa gerar um sistema de cuidados de saúde "a duas velocidades" (Kaplan e Litewka, 2008: 405; Spoonere Gotlieb, 2004: 641), ou seja, incrementando ainda mais as desigualdades em saúde já existentes (Hespanha, 2017). Hoje em dia, a disponibilidade de serviços remotos já permitirá aos/às cidadãos/ãs com um nível adequado de cuidados de saúde associar prestações convencionais com a telemedicina, incrementando a quantidade de serviços disponíveis e, eventualmente, também a qualidade do atendimento. Enquanto isso, os/as cidadãos/ãs que vivem em condição de isolamento social e/ou geográfico teriam que se contentar em colmatar a carência de serviços de saúde com a oportunidade de "ver" um médico em videoconferência, ou de aceder a prestações automáticas ou quase automáticas, como acontece no caso da telemonitorização e de muitas aplicações de saúde móvel. Portanto, a telemedicina apresenta-se como mais uma oportunidade de colocar a prática médica nas mãos das empresas privadas que fornecem estas prestações, contribuindo para tornar a saúde ainda mais um bem adquirível ao preço de mercado (Nunes, 2009).

Para evitar este risco, é necessário que a política de implementação da telemedicina no SNS seja acompanhada por uma visão crítica - alternativa à "visão inovadora" que resulta das orientações analisadas - e possivelmente inspirada numa perspetiva ética (Botrugno, 2017). Com base nesta perspetiva, a promoção da telemedicina deve ser calibrada não somente à luz das áreas prioritárias identificadas 
para o SNS - como foi feito, oportunamente, pelo Despacho n. ${ }^{\circ}$ 3571/2013 - , mas também quiçá à luz das implicações éticas e sociais subjacentes à adoção destes novos serviços na prática médica de rotina.

\section{Referências bibliográficas}

Adams, Samantha, e Maartje Niezen (2016), “Digital 'solutions' to unhealthy lifestyle 'problems': the construction of social and personal risks in the development of eCoaches", Health, Risk, \& Society, 17 (7-8), pp. 530-546.

Baszanger, Isabelle (1986), "Les maladies chroniques et leur ordre négocié", Revue Française de Sociologie, 27 (1), pp 3-27.

Bauer, Keith A. (2001), “Home-based telemedicine: a survey of ethical issues", Cambridge Quarterly Healthcare Ethics, 10 (2), pp. 137-146.

Bergrath, Sebastian, Daniel Rörtgen, Rolf Rossaint, Stefan K. Beckers, Harold Fischermann, Jorg Brokmann, Michael Czaplik, Marc Felzen, Marie-Thérèse Schneiders, e Max Skorning (2011), "Technical and organisational feasibility of a multifunctional telemedicine system in an emergency medical service: an observational study", Journal of Telemedicine and Telecare, 17, pp. 371-377.

Birns, Jonathan, Ayaj Bhalla, e Antony Rudd (2010), "Telestroke: a concept in practice", Age and Ageing, 39, pp. 666-667.

Blunt, Ian, Martin Bardsley, e Jennifer Dixon (2010), “Trends in emergency admissions in England 2004-2009", Nuffield Trust Report, disponível em: https://www.nuffieldtrust.org.uk/research/trends-in-emergency-admissions-in -england-2004-2009 (última consulta em fevereiro de 2020).

Botrugno, Carlo (2017), "Towards an ethics for telehealth", Nursing Ethics, 26 (2), pp. 357-367, disponível em: https://www.ncbi.nlm.nih.gov/pubmed/28502219 (última consulta em fevereiro de 2020).

Botrugno, Carlo (2018), "Telemedicine in daily practice: addressing legal challenges while waiting for a EU regulatory framework", Health, Policy \& Technology, 7 (2), pp. 131-136, disponível em: https://www.sciencedirect.com/science/article/abs /pii/S2211883718300856 (última consulta em fevereiro de 2020).

Botrugno, Carlo (2019), "Information technologies in healthcare: enhancing or dehumanising doctor-patient interaction?", Health Journal, DOI: $10.1177 / 1363459319891213$.

Botsis, Taxiarchis, e Gunnar Hartvigsen (2008), “Current status and future perspectives in telecare for elderly people suffering from chronic diseases", Journal of Telemedicine and Telecare, 7 (4), pp. 195-203.

Cartwright, Martin, Shashivadan Hirani, Lorna Rixon, Michelle Beynon, Helene Doll, Peter Bower, Martin Bardsley, Adam Steventon, Martin Knapp, Catherine Henderson, Anne Rogers, Caroline Sanders, Ray Fitzpatrick, James Barlow, e Stanton Newman (2013), "Effect of telehealth on quality of life and psychological outcomes over 12 months (Whole Systems Demonstrator telehealth questionnaire study): nested study of patient reported outcomes in a pragmatic, cluster randomised controlled trial", British Medical Journal, 346, f653. 
Cazzullo, Carlo, e Franco Poterzio (2007), “Paziente e medico: fenomenologia e prassi della relazione terapeutica", Roma, Edizioni Internazionali.

Chaudhry, Sarvat, Jennifer Mattera, Jeptha Curtis, John Spertus, Jeph Herrin, Zenqiu Lin, Christofer Phillips, Beth Hodshon, Lawton Cooper, e Harlan Krumholz (2010), "Telemonitoring in patients with heart failure", New England Journal of Medicine, 363, pp. 2301-2309.

Chiapperino, Luca, Marco Annoni, Paolo Maugeri, e Giuseppe Schiavone (2012), “What autonomy for telecare? An externalist approach", The American Journal of Bioethics, 12 (9), pp. 55-57.

Comissão Europeia (2004), Comunicação “Saúde em linha - melhorar os cuidados de saúde para os cidadãos europeus: plano de ação para um espaço europeu da saúde em linha", COM/2004/356.

Comissão Europeia (2007), Comunicação “Juntos para a saúde: uma abordagem estratégica para a UE (2008-2013)", COM/2007/630.

Comissão Europeia (2008), Comunicação “Sobre os benefícios da telemedicina para os doentes, os sistemas de saúde e a sociedade", COM/2008/689.

Comissão Europeia (2012), Comunicação "Plano de ação para a saúde em linha, 2012-2020 - cuidados de saúde inovadores para o século XXI", COM/2012/736.

Comissão Europeia (2013), Health Inequalities in the EU. Final Report of a Consortium, disponível em: https://ec.europa.eu/health//sites/health/files/social_determinants/ docs/healthinequalitiesineu_2013_en.pdf (última consulta em fevereiro de 2020).

Comissão Europeia (2014a), Green Paper on Mobile Health ("mHealth"), COM/2014/0219.

Comissão Europeia (2014b), Health Inequalities and eHealth. Report of the eHealth Stakeholder Group, disponível em: https://ec.europa.eu/digital-single-market/news/commissionpublishes-four-reports-ehealth-stakeholder-group (última consulta em fevereiro de 2020).

Conselho da Europa (2007), Recomendação CM/Rec(2007)16 do Comité de Ministros aos estados-membros, disponível em: https://www.coe.int/en/web/

freedom-expression/committee-of-ministers-adopted-texts/-/asset_publisher/aDX mrol0vvsU/content/recommendation-cm-rec-2007-16-of-the-committee-of-minister s-to-member-states-on-measures-to-promote-the-public-service-value-of-the-intern et?inheritRedirect=false\&desktop=true (última consulta em fevereiro de 2020).

Correia, Tiago (2013), “The interplay between managerialism and medical professionalism in hospital organisations from the doctors' perspective: a comparison of two distinctive medical units", Health Sociology Review, 22 (3), pp. 255-267, DOI: 10.5172/hesr.2013.22.3.255

Correia, Tiago, e Jean Louis Denis (2016), “Hybrid management, organizational configuration, and medical professionalism: evidence from the establishment of a clinical directorate in Portugal", BMC Health Services Research, 16 (S2), DOI: 10.1186/s12913-016-1398-2

Correia, Tiago, Graça Carapinheiro, Jorge Silva, e Joana Vieira (2015), “O sistema de saúde português no tempo da Troika: a experiência dos médicos", Lisboa, ISCTE-IUL / Ordem dos Médicos.

Cortes, Maria (2016), "Breve olhar sobre o estado da saúde em Portugal”, Sociologia, Problemas e Práticas, 80, pp. 117-143, disponível em: http://journals.openedition.org/spp/2117 (última consulta em fevereiro de 2020). 
EuroHealthNet (2011), consultado a 9 de abril de 2018 em: https://eurohealthnet.eu/sites/ eurohealthnet.eu/files/publications/Fact-Sheet-Digital-Divide-V2.pdf

FDA - Food and Drug Administration (2013), Mobile Medical Applications. Guidance for Industry and Food and Drug Administration Staff, consultado a 9 de abril de $2018 \mathrm{em:}$ http://www.fda.gov/downloads/MedicalDevices/.../UCM263366.pdf.

Gornall, Jonathan (2012), “Does telemedicine deserve the green light?", British Medical Journal, 345, e4622.

Gregoski, Mathew J., Martina Mueller, Alexey Vertegel, Aleksey Shaporev, Brenda B. Jackson, Ronja M. Frenzel, Sara M. Sprehn, e Frank A. Treiber (2012), "Development and validation of a smartphone heart rate acquisition application for health promotion and wellness telehealth applications", International Journal of Telemedicine and Applications, ID 696324, DOI: 10.1155/2012/696324.

Ha, Fong Jennifer, e Nancy Longnecker (2010), “Doctor-patient communication. A review", The Ochsner Journal, 10, pp. 38-43.

Hein, Matthew (2009), "Telemedicine: an important force in the transformation of healthcare", consultado a 9 de abril de $2018 \mathrm{em}$ : http://ita.doc.gov/td/health/telemedicine_2009.pdf.

Hespanha, Pedro (2008), "Políticas sociais: novas abordagens, novos desafios",Revista de Ciências Sociais, Universidade Federal do Ceará, 39 (1), pp. 5-15.

Hespanha, Pedro (2017), "As reformas dos sistemas de saúde na Europa do Sul: crises e alternativas", em Paulo Henrique Rodrigues e Isabela Santos (orgs.), Políticas e Riscos Sociais no Brasil e na Europa. Convergências e Divergências. São Paulo, Hucitec Editora, pp. 69-98.

Hespanha, Pedro, Silvia Ferreira, e Vanda Pacheco (2014), “O Estado Social, crise e reformas", em José Reis (org.), A Economia Política do Retrocesso. Crise, Causas e Objetivos, Coimbra, Almedina, pp. 189-281.

Kaplan, Bonnie, e Sergio Litewka (2008), "Ethical challenges of telemedicine and telehealth", Cambridge Quarterly of Healthcare Ethics, 17, pp. 401-416.

Kontos, Emily, Kelly D. Blake, Wen-Ying S. Chou, e AbbyPrestin (2014), “Predictors of eHealth usage: insights on the digital divide from the Health Information National Trends Survey 2012", Journal of Medical Internet Research, 16 (7): e 172, DOI: 10.2196/jmir.3117.

Kuhlmann, Ellen, Viola Burau, Tiago Correia, Roman Lewandowski, Christos Lionis, Mirko Noordegraaf, e Jose Repullo (2013)," 'A manager in the minds of doctors': a comparison of new modes of control in European hospitals", BMC Health Service Research, 13 (246), disponível em: https://doi.org/10.1186/1472-6963-13-246 (última consulta em fevereiro de 2020).

Latulippe, Karine, Christine Hamel, e Dominique Giroux (2017), “Social health inequalities and eHealth: a literature review with qualitative synthesis of theoretical and empirical studies", Journal of Medical Internet Research, 19 (4), e 136, DOI: 10.2196/jmir.6731.

Leh, Amy S. (2001), “Computer-mediated communication and social presence in a distance learning environment", International Journal of Educational Telecommunications, 7, pp. 109-128.

Lehoux, Pascale, Claude Sicotte, Jean-Louis Denis, Marc Berg, e André Lacroix (2002), "The theory of use behind telemedicine: how compatible with physicians' clinical routines?", Social Science and Medicine, 54 (6), pp. 889-904. 
Lehoux, Pascale (2008), "The duality of health technology in chronic illness: how designers envision our future", Chronic Illness, 4, pp. 85-97.

Lindberg, Birgitta, Carina Nilsson, Daniel Zotterman, Siv Söderberg, e Lisa Skär (2013), "Using information and communication technology in home care for communication between patients, family members, and healthcare professionals: a systematic review", International Journal of Telemedicine and Applications, vol. 2013, ID 461829, DOI: 10.1155/2013/461829.

Lindsay, Sally, Paul Bellaby, Simone Smith, e Rose Baker (2008), “Enabling healthy choices: is ICT the highway to health improvement?", Health, 12 (3), pp. 313-331, DOI: $10.1177 / 1363459308090051$.

Lupton, Deborah (2013), “The digitally engaged patient: self-monitoring and self-care in the digital era", Social Theory and Health, 11, pp. 256-270.

Matos, Rosa, Rui Santana, Rita Mendes, Patrícia Marques, e Ricardo Mestre (2014), Telemedicina em Portugal. Onde Estamos, Lisboa, Fundação Calouste Gulbenkian.

Matusitz, Jonathan, e Gerald-Mark Breen (2007), “Telemedicine: its effects on health communication", Health Communication and New Information Technologies, 21 (1), pp. 73-83.

McAuley, Andrew (2014), “Digital health interventions: widening access or widening inequalities?", Public Health, 128 (12), pp. 1118-1120, DOI: 10.1016/j.puhe.2014.10.008.

Milligan, Christine, Celia Roberts, e Maggie Mort (2011), “Telecare and older people: who cares where?", Social Science \& Medicine, 72 (3), pp. 347-354.

Ministério da Economia / IAPMEI (2000), Programa Operacional Sociedade da Informação, consultado a 1 de julho de 2015 em: http://www.iapmei.pt/index.php.

Ministério da Saúde (2000), Programa Operacional da Saúde - Saúde XXI, consultado a 1 de julho de 2015 em: http://www.portaldasaude.pt/portal/conteudos/ a+saude+em+portugal/ministerio/servicos/arquivo/saudexxi.htm.

Mort, Maggie, Celia Roberts, e Blanca Callén (2013), “Ageing with telecare: care or coercion in austerity?", Sociology of Health and Illness, 35 (6), pp. 799-812.

Mort, Maggie, Celia Roberts, Jeannette Pols, Mique lDomenech, e Ingunn Moser (2013), "Ethical implications of home telecare for older people: a framework derived from a multisited participative study", Health Expectations, 18, pp. 438-449.

Nagel, Saskia, e Hartmut Remmers (2012), , "Self-perception and self-determination in surveillance conditions", The American Journal of Bioethics, 12 (9), pp. 53-55.

Nicastri, Vincenzo (2012), “Scopi ed aspetti della telemedicina”, e-Health Care, 17, pp. 89-93.

Norman, Cameron D., e Harvey A. Skinner (2006), “eHealth literacy: essential skills for consumer health in a networked world"', Journal of Medical Internet Research, 8 (2), e9, DOI: 10.2196/jmir.8.2.e9.

Nunes, Arriscado J. (2009), "Velhos e novos desafios ao direito à justiça”, Revista Crítica de Ciências Sociais, 87, pp. 143-163.

OECD/EU (2014), Health at a Glance. Europe 2014, Paris, OECD Publishing, disponível em: https://www.oecd-ilibrary.org/docserver/health_glance_eur-2014-en.pdf?expires=1 581248780\&id=id\&accname=guest\&checksum=4BBF130F6E0E7562358FAF76218561 BA (última consulta em fevereiro de 2020).

OPSS - Observatório Português dos Sistemas de Saúde (2017), Relatório de Primavera 2017, Lisboa, OPSS, consultado a 8 de maio de 2018 em: http://www.opss.pt/sites/opss.pt/files/Relatorio_Primavera_2017.pdf 
OMS/CSDH - Organização Mundial da Saúde [Commission on Social Determinants of Health] (2003), Social Determinants of Health. The Solid Facts, disponível em: http://www.euro.who.int/_data/assets/pdf_file/0005/98438/e81384.pdf (última consulta em fevereiro de 2020).

OMS - Organização Mundial da Saúde (2010), Telemedicine. Opportunities and Developments in Member States. Report on the Second Global Survey on e-Health, Genebra, OMS, disponível em: http://www.who.int/goe/publications/goe_telemedicine_2010.pdf (última consulta em fevereiro de 2020).

Pols, Jeannette (2012), Care at Distance. On Closeness of Technology, Amesterdão, Amsterdam University Press.

Portaria n. ${ }^{\circ}$ 567, de 12 de junho de 2006, Diário da República, n. ${ }^{\circ}$ 113, I série-B.

Puga, Pedro, Gustavo Cardoso, Rita Espanha, e Sandro Mendonça (2009), "Telecommunications for the needy: how needed are they?", Informatica Economica, 13 (2), pp. 175-188.

Quesnel-Vallée, Amélie,Emilie Renahy, Tania Jenkins, e Helen Cerigo (2012), “Assessing barriers to health insurance and threats to equity in comparative perspective: the health insurance access database", BMC Health Services Research, 12 (107), DOI: 10.1186/1472-6963-12-107.

Raposo, Hélder (2009), “Risco e incerteza no pensamento biomédico: notas teóricas sobre o advento da quantificação e da prova experimental na medicina moderna", Análise Social, XLIV (193), pp. 747-765.

Research2Guidance (2013), “The mobile health global market report 2013-2017: the commercialisation of mHealth apps", vol. 3, consultado a 1 de setembro de $2015 \mathrm{em}$ : http://www.research2guidance.com/shop/index.php/downloadable/download/samp le/sample_id/262/.

Roback, Kerstin, e Almut Herzog (2003), “Home informatics in healthcare: assessment guidelines to keep up quality of care and avoid adverse effect", Technology and Healthcare, 11, pp. 195-207.

Rubies-Feijoo, Carles, Tomás Salas-Fernández, Francesc Moya-Olvera, e Joan Guanyabens-Calvet (2010), “Imagen médica, telemedicina y teleassistência médica", Medicina Clínica, 134 (supl. 1), pp. 56-62.

Santos, Boaventura de Sousa, e Sílvia Ferreira (2002), "A reforma do Estado-Providência entre globalizações conflituantes", em Pedro Hespanha e Graça Carapinheiro (orgs.), Risco Social e Incerteza. Pode o Estado Social Recuar Mais?, Porto, Afrontamento, pp. 177-225.

Spooner, Andrew S., e Edward M. Gotlieb (2004), "Telemedicine: pediatric applications", Pediatrics, 113: e639-643.

Steventon, Adam, e Martin Bardsley (2012), “The impact of telehealth on use of hospital care and mortality", Nuffield Trust Report, consultudo a 1 de novembro de 2014 em: http://www.nuffieldtrust.org.uk.

Webster, Andrew (2002), "Innovative health technologies and the social: redefining health, medicine and the body", Current Sociology, 50 (3), pp. 443-457.

Zissman, Keren, Izabella Lejbkowicz, e Ariel Miller (2012), “Telemedicine for multiple sclerosis patients: assessment using Health Value Compass", Multiple Sclerosis Journal, 18, pp. 472-479. 
Zuiderent, Teun, Marc Berg, e Britt Ross Winthereik (2003), “Talking about distributed communication and medicine: on bringing together remote and local actors", Human-Computer Interaction, 18 (1), pp. 171-180.

Zwijsen, Sandra A., Alistair R. Niemeijer, e Cees M. P. M. Hertogh (2011), “Ethics of using assistive technology in the care for community-dwelling elderly people: an overview of the literature", Aging \& Mental Health, 15, pp. 419-427.

Carlo Botrugno. Investigador e co-coordenador do Research Unit on Everyday Bioethics and Ethics of Science, Centro di Documentazione: L'altro diritto, Università di Firenze. E-mail: Carlo.botrugno@unifi.it

Joana R. Zózimo. Investigadora e co-coordenadora do Research Unit on Everyday Bioethics and Ethics of Science, Centro di Documentazione: L'altro diritto, Università di Firenze, e investigadora do SOCIUS/ISEG, Universidade de Lisboa. E-mail: joana.zozimo@gmail.com

Receção: 14 de maio de 2018 Aprovação: 08 de novembro de 2018 